\title{
A non-linear model of limit order book dynamics
}

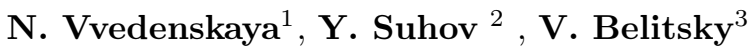

\begin{abstract}
This paper focuses on some simple models of limit order book dynamics which simulate market trading mechanisms. We start with a discrete time/space Markov process and then perform a re-scaling procedure leading to a deterministic dynamical system controlled by non-linear ODEs. This allows us to introduce approximants for the equilibrium distribution of the process represented by fixed points of deterministic dynamics.
\end{abstract}

\section{Introduction. The underlying Markov process}

In what follows, LOB stands for the limit order book, a trading mechanism adopted in many modern financial markets. For a detailed description of some common LOB models and their applications, see [1] and references therein. (Although our models differ in a number of aspects.) One of challenging problems is to determine factors attracting or repelling various market participants.

This paper explores a new approach to the analysis of LOB dynamics where the parameters of the original random (Markov) process are re-scaled, and a limiting dynamical system emerges, with a deterministic behavior described by a system of non-linear (ordinary) differential equations. A similar approach is commonly used in the literature on stochastic communication networks; see, e.g., the paper [2] and its sequels (in particular, [3]). In the current paper we consider a simplified model where a number of technically involved issues are absent. We also omit proofs, referring the reader to forthcoming publications beginning with [4].

The rationale for the models below is as follows. We consider a singlecommodity market where prices may be at one of $N$ distinct levels (say, $c_{1}<$ $c_{2}<\ldots<c_{N}$, although the exact meaning of these values is of no importance). The market is operating in discrete times $0, \delta, 2 \delta, \ldots$ At a given time $t \delta$, $t=0,1, \ldots$, there are $b_{i}(t)$ traders prepared to buy a unit of the commodity at price $c_{i}$ and $s_{i}(t)$ traders prepared to sell it at this price, which leads to vectors

$$
\boldsymbol{b}(t)=\left(b_{1}(t), \ldots, b_{N}(t)\right), \boldsymbol{s}(t)=\left(s_{1}(t), \ldots, s_{N}(t)\right) \in \mathbb{Z}_{+}^{N} .
$$

Here $\mathbb{Z}_{+}=\{0,1, \ldots\}$ stands for a non-negative integer half-lattice and $\mathbb{Z}_{+}^{N}$ for

\footnotetext{
${ }^{1}$ Institute for Information Transmission Problems, Russian Academy of Sciences, 19 Bol'shoi Karetny Per, GSP-4, Moscow 127994, RUSSIA; E-mail ndv@iitp.ru

${ }^{2}$ Institute for Information Transmission Problems, Russian Academy of Sciences, 19 Bol'shoi Karetny Per, GSP-4, Moscow 127994, RUSSIA; Instito de Matematica e Estatistica, Universidade de Sao Paulo, Caixa Postal 66281- CEP 05389-970 Sao Paulo, BRASIL; Statistical Laboratoty, DPMMS, University of Cambridge, Wilberforce Road, Cambridge CB3 0WB, UK; E-mail yms@statslab.cam.ac.uk

${ }^{3}$ Instito de Matematica e Estatistica, Universidade de Sao Paulo, Caixa Postal 66281- CEP 05389-970 Sao Paulo, BRASIL; E-mail belitsky@ime.usp.br
} 
the non-negative integer $N$-dimensional lattice orthant. The pair $(\boldsymbol{b}(t), \boldsymbol{s}(t))$ represent a state of a Markov process $\boldsymbol{U}(t)$ that will be the subject of our analysis.

If $b_{i}(t) \geq s_{i}(t)>0$ then each of the sellers gets a trade with probability $p_{\mathrm{T}} \in$ $(0,1)$ and leaves the market, together with his buyer companion. Therefore, both values $b_{i}(t)$ and $s_{i}(t)$ decrease by a random number $n=0,1, \ldots, s_{i}(t)$ with the binomial probability. A seller among $s_{i}-n$ who did not get the trade either (i) quits the market with probability $p_{\mathrm{Q}} \in(0,1)$ or (ii) moves to the price level $c_{i-1}$ with probability $p_{\mathrm{M}} \in(0,1)$ or (iv) remains at the same level with probability $1-p_{\mathrm{Q}}-p_{\mathrm{M}}$. (One can think that for this seller a random experiment is performed, with three outcomes.) Similarly, a buyer among $b_{i}(t)-n$ who did not get the trade quits the market with probability $p_{\mathrm{Q}} \in(0,1)$ or moves to the price level $c_{i+1}$ with probability $p_{\mathrm{M}} \in(0,1)$ or remains at the same level with probability $1-p_{\mathrm{Q}}-p_{\mathrm{M}}$. (Assuming that $p_{\mathrm{Q}}+p_{\mathrm{M}}<1$.)

Symmetrically, if $s_{i}(t) \geq b_{i}(t)>0$ then each of the buyers gets a trade with probability $p_{\mathrm{T}} \in(0,1)$ and leaves the market, together with his seller companion. The remaining traders at the price level $c_{i}$ proceed as above.

In addition, at every time point $t \delta$ a random number of new buyers arrive and position themselves at the price level $c_{1}$; it is distributed according to a Poisson law with mean $\Lambda_{\mathrm{b}}>0$. Similarly, at every time $t \delta$ a Poisson random number of sellers arrive and take a position at price level $c_{N}$; the mean value of this variable is $\Lambda_{\mathrm{s}}>0$.

All described events occur at each level independently. This generates the aforementioned Markov process $\{\boldsymbol{U}(t)\}$ with trajectories $\{(\boldsymbol{b}(t), \boldsymbol{s}(t))\}, t \in \mathbb{Z}_{+}$.

Theorem 1. $\forall$ values of parameters $\Lambda_{\mathrm{b} / \mathrm{s}}, p_{\mathrm{Q} / \mathrm{M}}$ and $p_{\mathrm{T}}$, the process $\{\boldsymbol{U}(t)\}$ is irreducible, aperiodic and positive recurrent. Therefore, it has a unique set of equilibrium probabilities $\pi=\left(\pi(\boldsymbol{b}, s): \boldsymbol{b}, \boldsymbol{s} \in \mathbb{Z}^{N}\right)$, and $\forall$ initial state $\boldsymbol{U}(0)$ (deterministic or random), the distribution of the random state $\boldsymbol{U}(t)$ at time $t$ converges weakly to $\pi$ as $t \rightarrow \infty$ :

$$
\lim _{t \rightarrow \infty} \mathbb{P}(\boldsymbol{U}(t)=(\boldsymbol{b}, \boldsymbol{s}))=\pi(\boldsymbol{b}, \boldsymbol{s})
$$

\section{Scaling limit}

The explicit form of the equilibrium distribution $\pi$ of process $\{\boldsymbol{U}(t)\}$ (and even probabilities of transitions $\left.(\boldsymbol{b}, \boldsymbol{s}) \mapsto\left(\boldsymbol{b}^{\prime}, \boldsymbol{s}^{\prime}\right)\right)$ are too cumbersome to work with. This fact makes it desirable to develop efficient methods of approximation.

In this paper we focus on one such method based on scaling the parameters of the process (including states and time-steps).

The re-scaling procedure is as follows: we set

$$
p_{\mathrm{T}}=\frac{\gamma}{L}, \quad p_{\mathrm{Q}}=\frac{\alpha_{\mathrm{Q}}}{L}, \quad p_{\mathrm{M}}=\frac{\alpha_{\mathrm{M}}}{L}, \quad \Lambda_{\mathrm{b}}=\frac{\lambda_{\mathrm{b}}}{L}, \quad \Lambda_{\mathrm{s}}=\frac{\lambda_{\mathrm{s}}}{L},
$$


where $\gamma>0, \alpha_{\mathrm{Q}}>0, \alpha_{\mathrm{M}}>0, \lambda_{\mathrm{b}}>0$ and $\lambda_{\mathrm{s}}>0$ are fixed and $L \rightarrow \infty$. In addition, we re-scale the states and the time: pictorially,

$$
x_{i} \sim \frac{b_{i}}{L}, y_{i} \sim \frac{s_{i}}{L}, \quad \tau \sim \frac{t \delta}{L} .
$$

Formally, denoting the Markov process generated for a given $L$ by $\boldsymbol{U}^{(L)}$, we consider the continuous-time process

$$
\boldsymbol{V}^{(L)}(\tau)=\frac{1}{L} \boldsymbol{U}^{(L)}(\lceil\tau L / \delta\rceil), \quad \tau \geq 0,
$$

where $\lceil a\rceil$ stands for the integer part of $a>0$.

Set: $\mathbb{R}_{+}=(0, \infty)$ (a positive half-line), then $\mathbb{R}_{+}^{N}$ is a positive orthant in $N$ dimensions. Suppose we are given a pair of vectors $(\boldsymbol{x}(0), \boldsymbol{y}(0)) \in \mathbb{R}_{+}^{N} \times \mathbb{R}_{+}^{N}$ where $\boldsymbol{x}(0)=\left(x_{1}(0), \ldots, x_{N}(0)\right), \boldsymbol{y}(0)=\left(y_{1}(0), \ldots, y_{N}(0)\right)$. Consider the following system of first-order ODEs for functions $x_{i}=x_{i}(\tau)$ and $y_{i}=y_{i}(\tau)$ where $\tau>0$ and $1 \leq i \leq N$ :

$$
\begin{aligned}
& \dot{x}_{1}=\lambda_{\mathrm{b}}-\left(\alpha_{\mathrm{Q}}+\alpha_{\mathrm{M}}\right) x_{1}-\gamma \min \left[x_{1}, y_{1}\right], \\
& \dot{x}_{i}=\alpha_{\mathrm{M}} x_{i-1}-\left(\alpha_{\mathrm{Q}}+\alpha_{\mathrm{M}}\right) x_{i}-\gamma \min \left[x_{i}, y_{i}\right], 1<i \leq N, \\
& \dot{y}_{i}=\alpha_{\mathrm{M}} y_{i+1}-\left(\alpha_{\mathrm{Q}}+\alpha_{\mathrm{M}}\right) y_{i}-\gamma \min \left[x_{i}, y_{i}\right], 1 \leq i<N, \\
& \dot{y}_{N}=\lambda_{\mathrm{s}}-\left(\alpha_{\mathrm{Q}}+\alpha_{\mathrm{M}}\right) y_{N}-\gamma \min \left[x_{N}, y_{N}\right],
\end{aligned}
$$

with the initial date $x_{i}(0), y_{i}(0), 1 \leq i \leq N$. The fixed point $\left(\boldsymbol{x}^{*}, \boldsymbol{y}^{*}\right)$ of system (51) has $\boldsymbol{x}^{*}=\left(x_{1}^{*}, \ldots, x_{N}^{*}\right)$ and $\boldsymbol{y}^{*}=\left(y_{1}^{*}, \ldots, y_{N}^{*}\right)$ where $x_{i}^{*}$ and $y_{i}^{*}$ give a solution to

$$
\begin{aligned}
& \lambda_{\mathrm{b}}=\left(\alpha_{\mathrm{Q}}+\alpha_{\mathrm{M}}\right) x_{1}^{*}+\gamma \min \left[x_{1}^{*}, y_{1}^{*}\right], \\
& \alpha_{\mathrm{M}} x_{i-1}^{*}=\left(\alpha_{\mathrm{Q}}+\alpha_{\mathrm{M}}\right) x_{i}^{*}+\gamma \min \left[x_{i}^{*}, y_{i}^{*}\right], 1<i \leq N, \\
& \alpha_{\mathrm{M}} y_{i+1}^{*}=\left(\alpha_{\mathrm{Q}}+\alpha_{\mathrm{M}}\right) y_{i}^{*}+\gamma \min \left[x_{i}^{*}, y_{i}^{*}\right], 1 \leq i<N, \\
& \lambda_{\mathrm{s}}=\left(\alpha_{\mathrm{Q}}+\alpha_{\mathrm{M}}\right) y_{N}^{*}+\gamma \min \left[x_{N}^{*}, y_{N}^{*}\right] .
\end{aligned}
$$

Both systems (5) and (6) are non-linear. However, the non-linearity 'disappears' at a local level which greatly simplifies the analysis of these systems.

In Theorems 2 and 3 below, we use the distance generated by the Euclidean norm in $\mathbb{R}^{N} \times \mathbb{R}^{N}$.

Theorem 2. $\forall$ initial date $(\boldsymbol{x}(0), \boldsymbol{y}(0)) \in \mathbb{R}_{+}^{N} \times \mathbb{R}_{+}^{N}$ there exists a unique solution $\{(\boldsymbol{x}(\tau), \boldsymbol{y}(\tau)), \tau>0\}$ to system (5). For this solution, $(\boldsymbol{x}(\tau), \boldsymbol{y}(\tau)) \in$ $\mathbb{R}_{+}^{N} \times \mathbb{R}_{+}^{N} \forall \tau>0$. As $\tau \rightarrow \infty$, the solution approaches a fixed point, which yields a unique solution to system (6):

$$
\operatorname{dist}\left[(\boldsymbol{x}(\tau), \boldsymbol{y}(\tau)),\left(\boldsymbol{x}^{*}, \boldsymbol{y}^{*}\right)\right] \rightarrow 0 \text {. }
$$


Theorem 3. Suppose that the re-scaled initial states $\frac{1}{L} \boldsymbol{U}(0)$ tend to vector $(\boldsymbol{x}(0), \boldsymbol{y}(0)) \in \mathbb{R}_{+}^{N} \times \mathbb{R}_{+}^{N}$ in probability: $\forall \epsilon>0$,

$$
\lim _{L \rightarrow \infty} \mathbb{P}\left(\operatorname{dist}\left[\frac{1}{L} \boldsymbol{U}(0),(\boldsymbol{x}(0), \boldsymbol{y}(0))\right] \geq \epsilon\right)=0 .
$$

Then, $\forall T>0$, the process $\left\{\boldsymbol{V}^{(L)}(\tau), \tau \in[0, T]\right\}$ converges in probability to the solution $\{(\boldsymbol{x}(\tau), \boldsymbol{y}(\tau)), 0 \leq \tau \leq T\}$. That is, $\forall \epsilon>0$,

$$
\lim _{L \rightarrow \infty} \mathbb{P}\left(\sup \left\{\operatorname{dist}\left[\boldsymbol{V}^{(L)}(\tau),(\boldsymbol{x}(\tau), \boldsymbol{y}(\tau))\right]: 0 \leq \tau \leq T\right\} \geq \epsilon\right)=0 .
$$

In particular, if $\boldsymbol{x}(0)=\boldsymbol{x}^{*}$ and $\boldsymbol{y}(0)=\boldsymbol{y}^{*}$ then

$$
\lim _{L \rightarrow \infty} \mathbb{P}\left(\sup \left\{\operatorname{dist}\left[\boldsymbol{V}^{(L)}(\tau),\left(\boldsymbol{x}^{*}, \boldsymbol{y}^{*}\right)\right]: 0 \leq \tau \leq T\right\} \geq \epsilon\right)=0 .
$$

Moreover, if process $\left\{\boldsymbol{U}(t), t \in \mathbb{Z}_{+}\right\}$is in equilibrium then Eqn (10) holds true.

\section{Fixed points in the scaling limit. Concluding remarks}

The approximation developed in Theorem 3 calls for an analysis of solutions to (6). As follows from the middle equations in (6),

Lemma 4. The fixed-point entries satisfy

$$
x_{1}^{*}>\ldots>x_{N}^{*} \text { and } y_{1}^{*}<\ldots<y_{N}^{*} .
$$

Consequently, the parameter space $\mathbb{R}_{+}^{5}$ formed by $\gamma, \alpha_{\mathrm{Q} / \mathrm{M}}$, and $\lambda_{\mathrm{b} / \mathrm{s}}$ is partitioned into open domains where one of the following generic patterns persists:

(i) $x_{N}^{*}>y_{N}^{*}$, (ii) $x_{1}^{*}<y_{1}^{*}$, and (iii) $x_{i}^{*}>y_{i}^{*}$ for $i=1, \ldots, \ell$ and $x_{i}^{*}<y_{i}^{*}$ for $i=\ell+1, \ldots, N$ where $1<\ell<N$. In each of these domains system (6) is linear.

Lemma 4 allows us to develop simple algorithms for calculating the fixed point $\left(\boldsymbol{x}^{*}, \boldsymbol{y}^{*}\right)$ and analyze the character of convergence in (77).

A particular algorithm for calculating $\left(\boldsymbol{x}^{*}, \boldsymbol{y}^{*}\right)$ is based on the following recursion. Set $x_{i}^{(0)}=0$ and let $y_{i}^{(0)}$ be the solution to the third and the forth equations (6) with $y_{N}^{(0)}=\lambda_{s} /\left(\alpha_{\mathrm{Q}}+\alpha_{\mathrm{M}}\right)$. Next, let $\left(\boldsymbol{x}^{(k)}, \boldsymbol{y}^{(k)}\right), k=1,2, \ldots$ be the solution to the system

$$
\begin{aligned}
& \lambda_{\mathrm{b}}=\left(\alpha_{\mathrm{Q}}+\alpha_{\mathrm{M}}\right) x_{1}^{(k)}+\gamma \min \left[x_{1}^{(k)}, y_{1}^{(k-1)}\right], \\
& \alpha_{\mathrm{M}} x_{i-1}^{(k)}=\left(\alpha_{\mathrm{Q}}+\alpha_{\mathrm{M}}\right) x_{i}^{(k)}+\gamma \min \left[x_{i}^{(k)}, y_{i}^{(k-1)}\right], 1<i \leq N, \\
& \alpha_{\mathrm{M}} y_{i+1}^{(k)}=\left(\alpha_{\mathrm{Q}}+\alpha_{\mathrm{M}}\right) y_{i}^{(k)}+\gamma \min \left[x_{i}^{(k)}, y_{i}^{(k)}\right], 1 \leq i<N, \\
& \lambda_{\mathrm{s}}=\left(\alpha_{\mathrm{Q}}+\alpha_{\mathrm{M}}\right) y_{N}^{(k)}+\gamma \min \left[x_{N}^{(k)}, y_{N}^{(k)}\right] .
\end{aligned}
$$


Lemma 5. The inequalities $x_{i}^{(k)}>x_{i}^{(k-1)}, y_{i}^{(k)}<y_{i}^{(k-1)}$ hold true $\forall i, k \geq 1$ and, values $x_{i}^{(k)}$ are uniformly bounded. Therefore, $\exists \lim _{k \rightarrow \infty} x_{i}^{(k)}$, and $\lim _{k \rightarrow \infty} y_{i}^{(k)}$ and these limits satisfy the system (6).

We conclude with the following remarks.

1. The current set-up admits straightforward generalisations to the case where parameters $\gamma$ and $\alpha_{\mathrm{Q} / \mathrm{M}}$ depend on $i, 0<i<N$ and on the trader type $(\mathrm{b} / \mathrm{s})$. A more complicated case emerges if parameters $\lambda_{\mathrm{b} / \mathrm{s}}$ become statedependent.

2. There are several forms of convergence for which the assertion in Theorem 3 holds true. The dynamical system (5) itself gives rise to a limiting process with interesting properties.

3. Another valid approximation for process $\boldsymbol{U}(t)$ is a diffusion approximation working on a different scale from that in (3).

These topics are subject to forthcoming research. See [4] and subsequent publications.

\section{References}

[1] I. Rosu. A Dynamic model of the limiting order book. //faculty.chicagoboot.edu/ioanid.rosu/research/limit.pdf.

[2] N.D. Vvedenskaya, R.L Dobrushin, F.I. Karpelevich. A queueing system with selection of the shortest of two queues: an asymptotical approach. Problems of Information Transmission, 32, 1996, 15-27.

[3] N.D. Vvedenskaya, Y.M. Suhov, Multy access system with many users: stability and metastability. Problems of Information Transmission, 43, 2007, No 3.

[4] N. Vvedenskaya, Y. Suhov, V. Belitsky. Non-linear models of the limit order book dynamics, I. In preparation (2011). 